\title{
Molecular diagnostics in the future: what will it look like and what are the challenges?
}

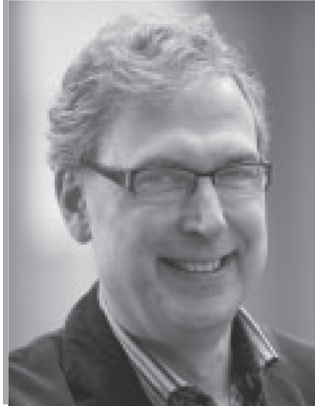

Hubert GM

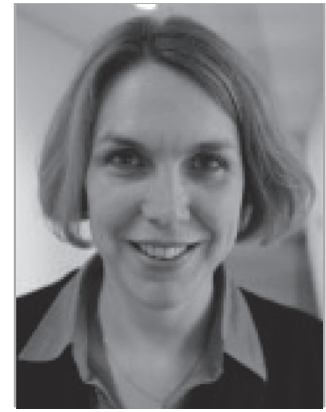

Coretta Cvan “...we have to critically look into the benefits of clinical diagnostics for patient care, as well as for infection control and prevention."

Niesters ${ }^{*, 1}$

Leer-Buter ${ }^{1}$

We have seen large changes in clinical virology laboratories over the last decades with the introduction of molecular diagnostic techniques. This may be for individual patient care or as a response to new appearing and discovered viruses, or to worldwide pandemics that have occurred. The rapid introduction of molecular assays against SARS and Middle East respiratory syndrome coronavirus, or against the influenza H1N1pdm09 strain, were made possible because it became relatively easy to characterize these viruses and to quickly develop assays based on real-time amplification techniques. However, these developments do not correspond to the daily practical problems a clinical laboratory has to deal with. The diversity of viral targets, the challenges in understanding the clinical implications of viral targets in several patient populations (children, immunocompromised patients), the need to detect low viral copies in for instance CNS infections, the turn around time to get a result that indeed has a clinical impact or the regulations that a laboratory has to keep up with, are the real challenges a laboratory has to deal with nowadays [1].

The future is known - results within 60 mins for each individual target
- however, there are some challenges we will most likely encounter. What are the challenges we most likely encounter?

It is obvious that the concept of samplein and result-out is limited for a few targets, like the blood-born viruses (HBV, HCV and HIV-1) in blood donor screening programs. The screening for Chlamydia trachomatis and soon, HPV, will be also introduced in several countries. The equipment needed for these assays are combined with US FDA- or Conformité Européenne/in vitro diagnostics-approved assays from diagnostic companies that have both the equipment and the assays. However, these are high volume screening programs and are of less of interest to the routine clinical diagnostic virology where a diverse group of assays are required for a diverse group of patients.

On the other hand, in recent decades diagnostic laboratories have invested an enormous amount of euros or dollars into a large range of equipment from different companies to enable them to deal with the diverse range of clinical samples and viral targets. Most, if not all of this equipment, was and is for research use only and is thus not FDA- or Conformité Européenne/in vitro diagnostics-approved.

\section{KEYWORDS}

- Accreditation and ISO guidelines

- droplet digital PCR

- middleware IT connectivity

- molecular diagnostics

- next-generation sequencing

- point-of-care tests

- very efficient processes

'University Medical Centre Groningen, The University of Groningen, Department of Medical Microbiology, Division of Clinical Virology, 
Asides from the equipment, there is a wide range of reagents, enzymes, primers and probes that can be purchased, which have to be combined within the regulatory boundaries, and with the research use only equipment. Therefore, it is obvious that there is, in general, a big difference in assays and equipment used for screening large, mostly negative and healthy populations (like the blood-born viruses), in contrast to the combinations of equipment and reagents needed for the so-called laboratory-developed tests. Moreover, we have also accepted that the equipment used for our laboratory-developed tests are not routinely linked electronically to a laboratory information system (LIS). Another problem is that equipment from different vendors cannot exchange information between each other using a common information technology (IT) language. Isolation and pipetting robots and real-time amplification equipment just do not 'talk' to one another. Diagnostic laboratories in general are struggling with the lack of connectivity of equipment and some have developed their own solutions. On the other hand, regulatory authorities have not challenged diagnostic companies to solve this lack of connectivity and exchange of information.

The virology world and the diagnostic laboratory are still changing and challenged. In large university hospitals, it is obvious that the number of viral targets continues to increase with the appearance of new viruses, such as HEV, Middle East respiratory syndrome coronavirus, human metapneumovirus, rhinovirus group $\mathrm{C}$ and influenza H7N7, and it is certain that this will continue. With the growing number of immunocompromised patients it is certain that the impact of diagnostic virology is also growing, because improved immune-suppressive regimens do increase virological diagnostic challenges. What is the impact of several viruses in these patient populations [1]?

There is also a tendency to get results faster to ensure that a clinical result, positive or negative, is made available as soon as possible. These so-called point-of-care tests are even available in a multiplex format (respiratory, gastroenteric) $[2,3]$. Again connectivity to a LIS is a necessity, and this issue remains to be solved. This fits into the picture of a very efficient diagnostic laboratory, in which the whole chain, from requests of assays and the diagnostic routing in the laboratory, up to getting the interpretation of the results, as well as the impact on for instance epidemiology, infection control and reporting to a health agency, are fully integrated into an IT solution. The electronic patient information system, the LIS, a middleware IT-connectivity solution and a reporting system, are all integrated and require no paper or pencil to rewrite data from one system into the other in the ideal very efficient world!

This very efficient world with an integrated total (IT) solution is in increasingly strong demand in the eyes of an accreditation agency using ISO guidelines like 15189:2012 [4]. Within these guidelines, risk assessment is the central theme; the laboratory has to put more effort into looking at critical steps in the whole process. This makes sense, as our clients (patients) are also demanding the best care, with no (or limited) risk. We should also look at our patients in a larger context. Patients are being moved within and between hospitals. Regional borders are no problem at all for viruses. Only by studying in detail the viral spread and polymorphisms will we understand how we can protect our most vulnerable patients from, for example, hospital-acquired infections $[5,6]$.

Diagnostics in general is often regarded merely as a cost factor. However, the benefits of diagnostics cannot be denied. When an outbreak of a new virus is around the corner, nobody argues against the benefit and costs of diagnostics, however, when we look into daily practice, our customers, or patients also are in need for the best care we can provide. Our patients turn to health professionals because they want a diagnosis, an explanation of what is going on and, if possible, a treatment. Good diagnostics is an essential part of this. A result with a short turnaround time where needed, performed at the highest standards, as we have agreed and as they are maintained by regulatory and accreditation agencies, should be the way to move forward. The benefits of diagnostics should be made more clear; patients not in isolation within a hospital, reduced hospital acquired infections, shorter period of stay within the hospital, optimal use of antiviral agents and antibiotics only if needed.

Looking backwards, we agree that the introduction of molecular tests have changed the clinical virology laboratory tremendously; we can generally detect and quantify all viral targets and we can rapidly develop new assays against new viral treats. The availability of proficiency panels, guidelines and accreditation processes have improved the overall quality of our work. 
However, some crucial steps still need to be worked on. One of them is the implementation of a common language to connect equipment from different vendors. Furthermore, we have to critically look into the benefits of clinical diagnostics for patient care, as well as for infection control and prevention. Viruses are continuously on the move between patients, between hospitals and do not stop at the borders of states or countries.

We are now facing two new developments that will have an impact on virology in general. The first is the development of next-generation sequencing technologies that make it possible to characterize viral genomes completely and to detect minor percentages of variants within a patient. The analysis of the enormous amount of data is a challenge for the near future, and an IT solution for integration within a diagnostic IT environment should be part of it [7]. The second development is the use of droplet digital PCR technologies that enable us to quantify viruses, their standards and minor populations within a clinical sample, more accurately $[8,9]$.

\section{References}

1 Caliendo AM, Gilbert DN, Ginocchio CC et al.; Infectious Diseases Society of America (IDSA). Better tests, better care: improved diagnostics for infectious diseases. Clin. Infect. Dis. 57(Suppl. 3), S139-S170 (2013).

2 Endimiani A, Hujer KM, Hujer AM et al. Are we ready for novel detection methods to treat respiratory pathogens in hospital-acquired pneumonia? Clin. Infect. Dis. 52(Suppl. 4), S373-S383 (2011).

3 Couturier MR, Barney T, Alger G et al. Evaluation of the FilmArray ${ }^{\circledR}$ Respiratory Panel for clinical use in a large children's

It is likely that both new technologies will find their way into the clinical diagnostic laboratory within a few years. They will provide more insight into the dynamic world in which we live together with the viruses that are around us. The known ones and the unknowns. The future will be a dynamic one full of new challenges!

\section{Financial \& competing interests disclosure}

HGM Niesters is an assessor for the Council of Accreditation in The Netherlands, and an Executive Board member of QCMD (Quality Control of Molecular Diagnostics, Glasgow, Scotland, United Kingdom). He has also been involved in development of a middleware solution for his Clinical Virology lab in UMCG, called FlowG (Flow Groningen), together with a commercial partner, Labhelp, Bladel, The Netherlands. The authors have no other relevant affiliations or financial involvement with any organization or entity with a financial interest in or financial conflict with the subject matter or materials discussed in the manuscript apart from those disclosed.

No writing assistance was utilized in the production of this manuscript.

hospital. J. Clin. Lab. Anal. 27(2), 148-154 (2013).

4 International Standard Organisation. www.iso.org

5 Rahamat-Langendoen JC, Lokate M, Schölvinck EH, Friedrich AW, Niesters HG. Rapid detection of a norovirus pseudo-outbreak by using real-time sequence based information. J. Clin. Virol. 58(1), 245-248 (2013).

6 Niesters HG, Rossen JW, van der Avoort H et al. Laboratory-based surveillance in the molecular era: the TYPENED model, a joint data-sharing platform for clinical and public health laboratories. Euro Surveill. 18(4), 20387 (2013).
7 Barzon L, Lavezzo E, Costanzi G, Franchin E, Toppo S, Palù G. Next-generation sequencing technologies in diagnostic virology. J. Clin. Virol. 58(2), 346-350 (2013).

8 Hayden RT, Gu Z, Ingersoll J et al. Comparison of droplet digital PCR to real-time PCR for quantitative detection of cytomegalovirus. J. Clin. Microbiol. 51(2), 540-546 (2013).

9 Henrich TJ, Gallien S, Li JZ, Pereyra F, Kuritzkes DR. Low-level detection and quantitation of cellular HIV-1 DNA and 2-LTR circles using droplet digital PCR. J. Virol. Methods 186(1-2), 68-72 (2012). 